\title{
Qigong as a mindful exercise intervention for people living with mental ill health
}

Chris Lloyd, Hector Tsang, Frank Deane

Background: Individuals with schizophrenia are more likely to be overweight or obese than the general population, and the associated health problems put them at a higher risk of death. Exercise is therefore an important non-pharmacological intervention that mental health workers can assist clients to engage in, and mindful exercise approaches may be particularly well-suited to this population. Content: This article describes the concept of qigong as a mindful exercise and looks at preliminary evidence suggesting that mindful exercise is of physical and emotional benefit to people with mental ill health. The authors propose an easy, standardized exercise protocol which clinicians can use and teach patient, and call for further research to explore its efficacy.

Conclusions: It is suggested that mindful exercise may be used as an intervention to assist people living with mental ill health to improve their community functioning and hence their recovery.

Key words: mental ill health mindful exercise qigong

Submitted 26 November 2008, send back for revisions 6 March 2009; accepted for publication following double-blind peer review 15 April 2009

\rceil ndividuals with schizophrenia have a higher risk of death than the general population, and as many as $75 \%$ of patients have a comorbid medical condition (Jones et al, 2004). Most deaths are a result of heart disease and cerebrovascular accidents (Anderson et al, 1991; Hennekens et al, 2005), and a multitude of factors are thought to contribute to these problems including higher rates of smoking, high fat-low fibre diets (Brown et al, 1999) and side effects of new antipsychotics which often induce weight gain (Wetterling and Müssigbrodt, 1999). In a community dwelling sample of 102 people with schizophrenia, it was found that $73 \%$ were either overweight or obese (McCreadie, 2003). An additional complication of obesity is its association with hypertension, type 2 diabetes (Dickerson et al, 2006), coronary heart disease, osteoarthritis, sleep apnoea and cancers. Distress from weight gain has also been shown to be related to antipsychotic medication non-compliance (Weiden and Olfson, 1995), lower quality of life (Allison et al, 2003) and social withdrawal in people with schizophrenia (Kurzthaler and Fleischhacker, 2001).

A Cochrane review of interventions to reduce weight gain in schizophrenia found 'modest' weight loss for both pharmacological and nonpharmacological (cognitive-behavioural) interventions (Faulkner et al, 2007). Non-pharmacological interventions appeared to be generally acceptable to participants, and included activities such as:

$\square$ Self-monitoring of weight, food and exercise

Modifying urges to overeat

Slowing the pace of eating

Exercise

Activity scheduling.

The review suggested the need to adopt a stepwise approach of incremental weight management interventions, and to support people to gradually increase their physical activity to 30-60 minutes of moderate activity most days of the week (Faulkner et al, 2007). It was also recommended that interventions that promote physical activity and changes in diet be implemented, and that such lifestyle interventions are likely to be within the skill sets of many mental health workers.

For many people, particularly those who are overweight or have been largely sedentary for long periods of time, gentle development of increased levels of exercise are needed. Mindful exercise approaches, such as yoga and qigong, have great potential in this regard and have the added potential benefit of increasing mindfulness. In view of its principle of integrating and harmonizing one's mind, breath, posture and movement ( $\mathrm{Ng}$ and Tsang, 2009), qigong may be a useful approach to adopt with people diagnosed with schizophrenia. In addition, this type
Chris Lloyd

is Senior Lecturer at the Queensland Centre for Mental Health Research and the School of Population Health, The University of Queensland;

Hector Tsang is Associate Professor at the Department of Rehabilitation Sciences, The Hong Kong Polytechic University; and Frank Deane is Head of Psychology and the Director of the Illawarra Institute of Mental Health University of Wollongong, Australia

Author for correspondence: $C$ Lloyd Email:Lloyd@onthenet. comau 
of intervention may be less threatening for people with schizophrenia because it is a relatively gentle approach to exercise compared to many traditional forms of exercise (e.g. aerobics, jogging, sports).

\section{AIMS}

This article describes qigong as an approach to mindful exercise, discussing the potential therapeutic mechanisms and its potential to relieve depression and anxiety among people with a psychotic disorder. An overview of mindful exercise and its effects on people with mental ill health is first given and the use of qigong is then explored.

\section{MINDFULNESS}

Mindfulness has been defined by Marlatt and Kristeller (1999: p68) as:

\section{'bringing one's complete attention to} the present experience on a moment-tomoment basis'.

Mindfulness as a treatment approach refers to elements in managing mental activities via exercising concentration and relaxation of the mind, so that individuals may be relieved from their perceived stressors ( $\mathrm{Li}$ and Sun, 1997; Tsang et al, 2002). There is growing research that suggests mindfulness has a range of positive effects on mental health (Baer, 2003). Mindfulness is a component of a range of effective psychological treatments for conditions such as borderline personality disorder, depression and relapse prevention for substance abuse (Baer, 2003). Praissman (2008) conducted a literature review of mindfulness in relation to its ability to reduce stress in a variety of populations and concluded that mindfulness is a safe, effective, integrative approach for reducing stress.

In a meta-analysis conducted by Grossman et al (2004), it was suggested that mindfulness as an intervention for a broad range of chronic disorders and problems (e.g. pain, cancer, heart disease, depression and anxiety) was a useful intervention. The authors suggested that the consistent and relatively strong level of effect sizes across very different types of samples indicated that mindfulness training might enhance general features of coping with distress and disability in everyday life.

\section{MINDFUL EXERCISE}

Mindful exercise has a focus on self-awareness and intrapersonal mind-body alignment, which is accompanied by low-to-moderate muscular exercise and non-judgmental meditation. It is different from most physical activities which focus on kinesthetic training and are relatively disconnected with the mind.
Mindful exercise includes introspective meditation, proprioceptive awareness of bodily movement, breath-centering techniques, anatomic alignment postures, and contemplation of energy-centric flow (Forge, 2005). It originated from two ancient oriental exercises, qigong and yoga, which share the common feature of mind, body and spirit integration.

Mindful exercise has not been a focus of interventions for people with severe mental ill health such as schizophrenia, although individuals living with mental ill health have often been attracted to mindful activities such as meditation and yoga. Yoga is arguably better known in Western societies and people with serious mental ill health frequently report the use and benefits of meditation and yoga. For example, in a sample of 157 individuals with 'serious mental illness' who were asked about their use of alternative health-care practices, $43 \%$ indicated they used meditation and 20\% did yoga (Russinova et al, 2002). For those who did meditation, the area of greatest perceived benefit was increased emotional calmness $(77 \%)$ but respondents also reported improved physical health (27\%). The main perceived benefit of yoga was also increased emotional calmness, endorsed by $58 \%$ of respondents, followed by improved physical health (Russinova et al, 2002)

However, there is very limited research on the effects of mindful exercise as adjuncts in mental health rehabilitation, and it is currently not clear whether the mindfulness components of interventions such as yoga are required for improvements in outcomes. A single-blind randomized trial compared yoga with physical exercise therapy as treatment adjuncts for people with schizophrenia over a 4month period (Duraiswamy et al, 2007). Physical exercise involved brisk walking, jogging and various exercises in both standing and sitting positions. Only 41 of the original 61 participants had data available at the 4-month follow-up. However, participants in the yoga group had significantly less psychopathology, better social and occupational functioning and better quality of life at follow-up compared to those in the physical exercise group (Duraiswamy et al, 2007). It is important to note that the yoga intervention explicitly excluded meditation, which is one approach to increasing mindfulness. However, the authors concluded that yoga therapy was useful as an add-on adjunct in the treatment of people with schizophrenia, but noted the need for longer-term follow-up.

\section{QIGONG AS AN INTERVENTION}

Qigong is an ancient art of self-healing which originated from mainland China in around 17661123 BC (Zhang, 1990). Qi means vital energy and gong means discipline. Qigong is mainly composed 
of five elements (Lin et al, 1995):

avisualization

Meditation

- Relaxation

$\square$ Deep breathing

- Target qi circulation.

Qigong focuses on the balance of yin and yang, as well as smoothing the circulation of $q i$ inside the body. Such an exercise is for yang sheng (regi-. ment) and bao jian (maintenance of health); these health concepts are well accepted in Chinese culture. Qigong has three main features:

Q Postures and movement

State of mind

Breathing.

Although yoga and tai chi share similar fea* tures with qigong, there are major differences in the form of physical movement and the physical demand involved. Yoga focuses on body flexibility and stretching (Feuerstein. 1996) and tai chi has an emphasis on lower limb strength and coordination (Tsang and Hui-Chan, 2005). In contrast, the specific form of qigong introduced in this article has eight distinctive forms of movements, which are illustrated below. Qigong can be conducted in static (sitting, lying or standing) or dynamic (moving) styles. Qigong can be practised alone as a homebased activity or in groups with the potential added benefits of social support (Xin et al, 2007). The aim of practising qigong is to cultivate $q i$ to help the individual stay healthy. In China, health and longevity are determined by strength, balance and cultivation of the three treasures:

\section{Jing (essence)}

Qi (energy)

ahen (spirit).

Qigong focuses on these three treasures to represent a holistic view of the human being. Popular forms of qigong include the Eight-Section Brocades (baduanjin), Guolin qigong, Yi Jin Jing and Chan Mi spine exercise, which are essentially for health promotion. The Eight-Section Brocades (Tsang et al, 2008) is a form of health-promoting Chinese qigong that can be easily taught and learnt. It is available in two training methods: The Sitting-Style Eight-Section Brocades and the Standing-Style Eight-Section Brocades. This means that it can be practised by people who have poor standing balance or are wheelchair-bound, for example. It is suggested here that the Eight-Section Brocades is a suitable form of mindful exercise in mental health settings because it is easy to learn and practice, it has the three elements of qigong, and can be practised in both sitting and standing. Description of the eight movement routine is given in Table 1 .

Qigong has been found to be beneficial for people experiencing a range of physical health prob-

lems, such as fibromyalgia (Austin et al, 2003), osteoporosis (Chen et al, 2006), hypertension (Cheung et al, 2005), cardiac functioning in older people (Du et al, 2006), coronary artery disease (Stenlund et al, 2005) and muscular dystrophy (Wenneberg et al, 2004). Ng and Tsang (2009) suggested that the ultimate aim of qigong is to enhance the self-healing ability when recovering from diseases and/or the self-defence ability to guard against diseases.

\section{USE OF QIGONG TO RELIEVE DEPRESSION AND ANXIETY}

As well as use for benefits on physical health, qigong has also been used with positive results for mental health problems, such as depression and anxiety (Tsang et al, 2003; 2006; Chou et al, 2004; Siu et al, 2007; Johansson et al, 2008). A recent review of 12 randomized controlled trials (RCTs) on the use of both mindful and non-mindful exercises for people with depression identified six of the 12 RCTs applied physical exercise with mindful elements (Tsang et al, 2008). Participants ranged from middle-aged to older-age subjects, with a mean sample size of 42 . The six RCTs survived basic quality assessment such as random allocation and intention to treat analyses. The physical exercises included qigong, yoga and tai chi. All of the studies reported positive short-term results on depression using the Hamilton Rating Scale for Depression or the Geriatric Depression Scale although long-term effects remained undetermined. Similarly, qigong has been reported to reduce anxiety symptoms and neuroticism (Tang et al, 1995; Leung and Singhal, 2004). Tsang et al (2002) suggested that older people who experience chronic physical illnesses are likely to have depression because of their disabilities and reduced psychosocial resources. RCTs found

\section{Table 1.}

Eight-Section Brocades (Tsang et al, 2008)

1. Prop up the sky with both hands to regulate
the triple warmer

2. Draw a bow on both sides like shooting a vulture

3. Raise single arm to regulate spleen and stomach

4. Look back to treat five strains and seven impairments

5. Sway head and buttocks to expel heart-fire

6. Pull toes with both hands to reinforce kidney and waist

7. Clench fists and look with eyes wide open to build up strength and stamina

8. Rise and fall on tiptoes to dispel all diseases
兩手托天理三焦

左右開弓似射鵰

調理脾胃須單舉

五勞七傷往後燋

搖頭㨢尾去心火

兩手攀足固腎腰

攢拳怒目增氣力

背後七顛百病消 
that, having received 12-16 weeks of regular qigong exercise, older adults experienced fewer perceived limitations and had a higher sense of mastery and self-efficacy compared to those in the control group receiving newspaper discussion as the control activity (Tsang et al, 2003; 2006). Moreover, participants experienced better social support and interpersonal relationships and these favourable experiences helped relieve depression.

\section{Mechanisms of action}

While there is emerging evidence to show that the effects of qigong improve psychological and physical wellbeing, the underlying psychobiological mechanisms remain elusive. Recent reviews (Chow and Tsang, 2007; $\mathrm{Ng}$ and Tsang, 2009; Tsang and Fung, 2009) suggest that qigong seems to improve human physical and psychological functions via neuroendocrine modulation and thus reduces stressrelated hormones, blood pressure and anxiety levels (Lee et al, 2001; Lee et al, 2004). Tsang and Fung (2009) suggest three neurophysiological mechanisms to explain the anti-depressive effect of qigong, based on the neurotransmitter, neuroendocrine and neurotropic perspectives. These hypotheses are based on review of extant literature and findings by Tsang et al $(2003 ; 2006)$ which suggest the pattern of therapeutic progress is similar to the action of serotonin selective reuptake inhibitors (SSRI), a classical anti-depressive agent (Stahl, 2000). These mechanisms are that qigong:

Increases the level of monoamine neurotransmitters in the brain

Reduces stressful signals to the limbic system and thus reduces the hypothalamus pituitary adrenal axis activity resulting in the reduction of release in adrenal glucocorticoid

Reduces stress leading to up-regulation of the brain-derived neurotrophic factor and down-regulation of adrenal cortisol; the combined result of which is the promotion of neurogenesis in the hippocampus.

The psychobiological mechanism of health qigong may involve down regulation of the activity of the sympathetic nervous system, modulation of the immune system either by changing the number of immune cell types or by improving the functioning of specific immune cells, regulation of the metabolism of blood lipids, improvement of circulation through improving the contracting and relaxation abilities of the heart, improvement of oxygenation by improvement in tidal volume and minimizing anatomic dead space. With regard to its anxiety reducing effects, it is suggested that qigong deepens the brain activity to a meditative and relaxed state, which then reduces anxiety and negative mood states (Lee et al, 2001).
The potential benefits of qigong therefore appear to be in the domains of physical functioning (e.g. muscle relaxation), emotional functioning (e.g. increased emotional stability, decreased stress), cognitive functioning (e.g. improved concentration, increased self-esteem), social functioning (e.g. decreased social isolation), and general functioning (e.g. increased sense of wellbeing).

\section{APPLICATION AMONG PEOPLE LIVING WITH MENTAL ILL HEALTH}

Rehabilitation professionals such as occupational therapists and physical therapists may consider applying this simple protocol of mindful exercise in their settings. Practitioners who intend to use this new approach for mental health rehabilitation are encouraged to contact the second author for details. Based on the authors' experience of using qigong for older people with depression, standardizing the mindful exercise protocol is the recommended first step. The protocol is simple and easy to use and is likely to be applicable in day programmes. Clinicians who wish to apply this mindful exercise can first of all learn and practise it for themselves using a video available from the second author. A train-the-trainer module for practitioners who would like to teach this to clients is now being developed. It is suggested that after completing the two online training programmes, clinicians will have adequate competence to implement it at their own settings.

A second step is to conduct controlled clinical studies to study the effects of the qigong protocol on people seen in mental health rehabilitation contexts. Primary outcomes should include reductions in perceived stress in participants' living environments, relief of depressive and anxiety symptoms, and improvements in psychological wellbeing. Subsequent studies should assess more long-term outcomes, such as community functioning and quality of life.

\section{CONCLUSIONS}

This article has reviewed the literature concerning the physical activity patterns and health problems of people living with mental ill health. Non-pharmacological interventions appear to be generally acceptable to participants. It has been shown that people with mental health problems have often been attracted to mindful activities such as meditation and yoga. Mindful exercise has a focus on self-awareness and intrapersonal mind-body alignment. One form of mindful exercise, qigong, involves postures and movement, state of mind and breathing. The Eight-Section Brocade is a 
form of health promoting qigong that can be easily taught and learnt. It has been found that qigong is beneficial to physical functioning, emotional functioning, cognitive functioning, and social functioning. It is suggested here that standardizing the mindful exercise protocol is needed, as is more research on the benefits for people living with mental ill health. IJTR

Conflict of interest:

Allison DB, Mackell JA, McDonnell DD (2003) The impact of weight gain on quality of life among persons with schizophrenia. Psychiatr Serv 54: 565-7

Anderson C, Connelly J, Johnstone EC, Owens DGG (1991) Cause of death. Br J Psychiatry 159: 30-3

Austin JA, Berman BM, Baussell B, Lee WL, Hochberg M, Forys KL (2003) The efficacy of mindfulness meditation plus Qigong movement therapy in the treatment of fibromyalgia: A randomized controlled trial. J Rheumatol 30: 2257-62

Baer RA (2003) Mindfulness training as a clinical intervention: A conceptual and empirical review. Clinical Psychology: Science and Practice 10: 125-43

Brown S, Birtwhistle J, Roe L, Thompson C (1999) The unhealthy lifestyle of people with schizophrenia. Psychol Med 29: 697-701

Chen HH, Yeh ML, Lee FY (2006) The effects of Baduanjin qigong in the prevention of bone loss for middle-aged women. Am J Chinese Med 34: 741-7

Cheung BM, Lo JL, Fong DY et al (2005) Randomised controlled trial of qigong in the treatment of mild essential hypertension. J Hum Hypertens 19: 697-704

Chou KL, Lee PWH, Yu ECS, Macfarlane D, Cheng YH, Chan SSC, Chi I (2004) Effect of Tai Chi on depressive symptoms amongst Chinese older patients with depressive disorders: a randomized clinical trial. Int $J$ Geriatr Psychiatry 19(11): 1105-7

Chow YWY, Tsang HWH (2007) Biopsychosocial effects of Qigong as a mindful exercise towards individuals with anxiety disorders: A speculative review. J Altern Complement Med 13: $831-40$

Dickerson FB, Brown CH, Kreyenbuhl JA, Fang L, Goldberg, RW, Wohlheiter K, Dixon LB (2006) Obesity among individuals with serious mental illness. Acta Psychiatr Scand 113: $306-13$

Du SW, Cheng QL, Wang H et al (2006) The effects of health qigong yi jin jing on cardiac function of the elderly. Chinese Journal of Sports Med 25: 721-2

Duraiswamy G, Thirthalli J, Nagendra HR, Gangadhar BN (2007) Yoga therapy as an add-on treatment in the management of patients with schizophrenia- a randomized controlled trial. Acta Psychiatr Scand 116: 226-32

Hennekens CH, Hennekens AR, Hollar D, Casey DE (2005) Schizophrenia and increased risks of cardiovascular disease. Am Heart J 150: 115-21

Faulkner G, Cohn T, Remington G (2007) Interventions to reduce weight gain in schizophrenia. Cochrane Database Syst Rev 24(1): CD005148

Forge RL (2005) Aligning mind and body: Exploring the disciplines of mindful exercise. ACSM's Health and Fitness $J$ 9: 7-14

Feuerstein G (1996) The Shambhala Guide to Yoga. Shambhala Publications, Boston: 26

Grossman P, Niemann L, Schmidt S, Walach H (2004) Mindfulness-based stress reduction and health benefits: a meta-analysis. J Psychosom Res 57: 35-43

Johansson M, Hassmén P, Jouper J (2008) Acute effects of qigong exercise on mood and anxiety. Int $J$ Stress Manag 15(2): 199-207

Jones DR, Macais C, Barreira PJ et al (2004) Prevalence, severity and co-occurrence of chronic physical health problems of persons with serious mental illness. Psychiatr Sen 55: $1250-7$

Kurzthaler I, Fleischhacker WW (2001) The clinical implications of weight gain in schizophrenia. $J$ Clin Psychiatry 62: $32-7$

Lee MS, Kang CW, Lim HJ, Lee MS (2004) Effects of qitraining on anxiety and plasma concentrations of cortisol,
$\mathrm{ACTH}$, and aldosterone: A randomized placebo-controlled pilot study. Stress and Health 20: 243-8

Lee MS, Huh HJ, Kim BG et al (2001) Psychoneuroimmunological effects of Qi-therapy: Preliminary study on the changes of level of anxiety, mood cortisol and melatonin and cellular function of neutrophil and natural killer cells. Stress and Health 17: 17-24

Leung Y, Singhal A (2004) An examination of the relationship between qigong meditation and personality. Soc Behav Pers 32: 313-20

Li XJ, Sun WY (1997) Chi Kung: Increase Your Energy, Improve Your Health. Sterling Publishing Company, New York

Lin TY, Tseng WS, Yeh EK (1995) Chinese Societies and Mental Health. Oxford University Press, New York

Marlatt GA, Kristeller JL (1999) Mindfulness and meditation. In: Miller WR. ed. Integrating spirituality into treatment. American Psychological Association, Washington DC: $67-84$

McCreadie RG (2003) Diet, smoking and cardiovascular risk on people with schizophrenia. Br J Psychiatry 183: 534-9

$\mathrm{Ng} \mathrm{B}$, Tsang HWH (2009) Psychophysiological Outcomes of Health Qigong for Chronic Health Conditions: A Systematic Review. Psychophysiology 46: 257-69

Praissman S (2008) Mindfulness-based stress reduction: A literature review and clinician's guide. J Am Acad Nurse Pract 20: $212-16$

Russinova Z, Wewiorski NJ, Cash D (2002) Use of alternative health care practices by persons with serious mental illness: Perceived benefits. Am J of Public Health 92: 1600-3 Siu JY, Sung H, Lee W (2007) Qigong practice among chronically ill patients during the SARS outbreak. $J$ Clin Nurs 16(4): 769-76

Stahl SM (2000) Placebo-controlled comparison of the selective serotonin reuptake inhibitors citalopram and sertraline. Biol Psychiatry: 48: 894-901

Stenlund T, Lindström B, Granlund M, Burell G (2005) Cardiac rehabilitation for the elderly: Qi Gong and group discussions. Eur J Cardiovasc Prev Rehabil 12: 5-11

Tang CM, Wei X, Zhang JP et al (1995) Effects of qigong on personality-A longitudinal study. Chinese Journal of Behavioral Medical Science 4: 15-18

Tsang HWH, Cheung L, Lak DCC (2002) Qigong as a psychosocial intervention for depressed elderly with chronic physical illnesses. Int $J$ Geriatr Psychiatry 17: 1146-54

Tsang HWH, Fung KMT (2009) Neurobiological and psychological mechanisms underlying the anti-depressive effect of Qigong exercise.J Health Psychol: In press

Tsang WWN, Hui-Chan CWY (2005) Comparison of muscle torque, balance, and confidence in older Tai Chi and healthy adults. Med Sci Sports Exerc 37: 280-9

Tsang HWH, Mok CK, Au Yeung YTA, Chan SYC (2003) The effect of Qigong on general and psychosocial health of elderly with chronic physical illnesses: A randomized clinical trial. Int $J$ Geriatr Psychiatry 18: 441-9

Tsang HWH, Fung KMT, Chan ASM et al (2006) Effect of a

\section{KEY POINTS}

- Mindful exercise has a focus on self-awareness and intrapersonal mind-body alignment

- Mindful exercise has not been a focus of intervention for people with severe mental ill health but there is emerging evidence highlighting its benefits for mental as well as physical health.

- This article discusses the role of qigong, a mindful exercise, as an adjunct treatment for severe mental health problems, and proposes the Eight-Section Brocade form as a suitable method for clinicians to use and teach patients.

- Studies should assess more long-term outcomes, such as community functioning and quality of life, of this and other mindful exercises. 
qigong exercise programme on elderly with depression. Int $J$ Geriatr Psychiatry 21:890-7

Tsang HWH, Chan EP, Cheung WM (2008) Effects of mindful vs non-mindful exercise interventions on people with depression: A systematic review. Br J Clin Psychol 47: 303-22

Wenneberg S, Gunnarsson LG, Ahlström G (2004) Using

a novel exercise programme for patients with muscular

dystrophy. Part II: A quantitative study. Disabil Rehabil 26: 595-602

Wetterling T, Müssigbrodt HE (1999) Weight gain: Side effects of atypical neuroleptics? J Clin Psychopharmacol 19: $316-21$

Weiden PJ, Olfson M (1995) Cost of relapse in schizophrenia. Schizophrenia Bull 21: 419-29

Xin L, Miller YD, Brown WJ (2007) A qualitative review of the role of Qigong in the management of diabetes. J Altern Complement Med 13: 427-3

Zhang E (1990) Chinese Qigong in a Practical EnglishChinese Library of TCM. Publishing House of Shangha College of Traditional Chinese Medicine, Shanghai
The promise and challenge of research in 'mindful' exercise: Meditative movement as a category of exercise

In this article, Lloyd et al provide yet another potential contribution to the growing body of research that addresses the health and mental wellbeing benefits of meditative movement (e.g. qigong, tai chi, yoga). Jahnke et al (2009, unpublished observations) recently completed a review of all of the randomized controlled trials (RCTs) conducted on health benefits of qigong and tai chi through to December 2007. By that date, 67 RCTs had been published and another 11 RCTs have since appeared in press. In reviewing these studies, it appears that not all are of the quality or required power to confirm effects, but the magnitude of evidence for the potential of these practices across many domains of health is impressive.

Robust findings are accruing in the areas of cardiopulmonary health, physical function, quality of life and other patient reported outcomes, falls prevention and balance, and even bone health.
Research specifically targeting more severe mental illness is sparse, but in research with populations experiencing anxiety or depression, benefits of qigong or tai chi are fairly consistently, significantly demonstrated. This current article suggesting the extension of research to a more severely debilitated mentally ill population is an important contribution to the literature.

In reviewing the extant body of evidence, we have found that very few studies discuss or assess the possible mechanisms of action. First of all, rarely do the descriptions of intervention protocols outline the key components of posture or movement, the mindful aspects, and the focus on the breath. Similarly, the link between these aspects of exercise, breathing and mindfulness, and the possible associated neurohormonal changes of the relaxation response (and possibly, as we grow to understand more about it, the 'qi' effects), are rarely addressed in the RCTs of meditative movement.

In the study proposed in this article, the standardization of the protocol is expected to insure a degree of intervention fidelity. The obvious expertise and dedication to qigong as a whole-person wellness regimen is expressed in the rich description of the tenets, practice and experience of qigong. It is expected that there will be a set of measures to confirm that the mindful state and breathing are practised by participants in the study as taught. Of particular interest is the care that has gone into reviewing the possible mechanisms of action. The proposed study will make a most profound extension of our understanding of effects on mental health if some of the key biomarkers are assessed along the way for the population under study.

The field of meditative movement (or mindful exercise) is at the brink of being able to extend our understanding past parochial applications of single practices, into an era of capturing what 'works' when one is 'harmonizing one's mind, breath, posture and movement'. As we begin to treat mindful or meditative movement as a category of exercise, and assure that the key components are implemented regardless of specific exercises chosen, establishing the health benefits across these practices will develop exponentially (as has been done with aerobic exercise as a category). Studies that define these components, and drill deeper to assess which of these are responsible for achieving results, are going to move the field forward. This proposed study is poised to do just that, and the results will be eagerly awaited.

\section{Linda K Larkey}

Professor, Scottsdale Healthcare Chair of Biobehavioral Oncology Research,

College of Nursing and Health

Innovation,

Arizona State University, USA

Larkey LK, Jahnke R, Etnier J, Gonzalez J (2009) Meditative Movement as a category of exercise: Implications for research. JPhys Act Health 6(2): 230-8

Rogers CE, Larkey LK, Keller C (2009) A review of clinical trials of Tai Chi and Qigong in older adults, West I Nurs Res 31: $245-279$
Curiously, as we have fairly recently entered a new millennium that in many ways has been focused on new technological and scientific advancements, clinical research and practice has turned to ancient traditions such as meditation, yoga, exercise, prayer, and in this article, qigong, to improve wellbeing and both physical and psychological functioning among our healthy and unhealthy populations. While we live in a world that is changing so rapidly as a result of improved computer, telecommunications, travel and medical technology, we turn to ancient wisdom and techniques to help us solve or cope with our contemporary struggles.
During the past decade, there has been an explosion in high quality research using the best contemporary methodological approaches such as randomized clinical trials to examine the potential benefits of many of the ancient healing traditions (see reviews by Koenig et al, 2001; Powell et al, 2003; Masters et al,
2006; Pargament, 2007; Plante and Thoresen, 2007; Plante, 2009). The psychiatric and psychological professional communities have embraced mindfulness interventions and have incorporated these techniques into many modern clinical interventions (e.g. Kabat-Zinn, 2003; Hayes, 2005; Hayes et al, 2004). Research 
findings are very promising. As well articulated in this article, mindfulness exercise, such as qigong, has been carefully evaluated in a number of well conducted randomized clinical trials used for those who suffer from significant mental illness such as schizophrenia and findings thus far seem highly encouraging.

A variety of factors may explain why mandolines exercise can help those who suffer from mental illness feel better. Biological, psychological, social, and even placebo effects may play an active role in healing (Plante, 2009). Future research will need to tease these issues apart to develop intervention programmes and approaches that maximize benefits and minimize costs and risks

One area for caution in this research is that many researchers and practitioners may perhaps maintain vested interests in the clinical results, and may wish to demonstrate that these ancient traditions-which may be important in their personal and professional lives - can be used to treat whatever ails others. Objective scientific neutrality can sometimes be hard to come by in research and practice in this area (Sloan et al, 1999). Therefore, perhaps extra efforts to conduct the most rigorous scientific research and offer interventions that are based on the best available science should be encouraged and underscored. Ethical precautions and considerations are also critical.

However, as this article has articulated, ancient traditions involving mindfulness exercise such as qigong appear to be helpful to people struggling with mental health as well as physical health challenges and disabilities These low cost, safe, and often enjoyable interventions that have been with us for centuries appear to help manage contemporary problems for modern man. It is encouraging indeed.

\section{Thomas G Plante}

Adjunct Clinical Professor, of Psychiatry and Behavioral Sciences, Stanford University School of Medicine; and Professor of Psychology,

Santa Clara University, Alumni Science 203,

500 El Camino Real,

Santa Clara, CA 95053-0333, USA

Hayes SC, Follete VM, Linehan MM (2004) Mindfulness and acceptance: Expanding the cognitive-behavioral tradition. Guilford, New York

Hayes SC (2005) Get out of your mind and into your life: The new acceptance and commitment therapy. New Harbinge, Oakland, CA

Kabat-Zinn I (2003) Mindfulness-based interventions in context: Past, present. and future. Clinical Psychology: Resrarch and Practice 10: 144-56

Koenig HG, McCullough ME, Larson DB (2001) Handbook of religion and health. Oxford Publishing, New York

Masters KS, Spielmans GI, Goodson JT (2006) Are there demonstrable effects of distant intercessory prayer? A meta-analytic review. Ann Behav Med 32: 337-42

Pargament $\mathrm{Kl}$ (2007) Spiritually integrated psychotherapy: Understanding and dddressing the sacred. Guilford, New York

Plante TG, Thoresen CE, eds (2007) Spirit, science and health: How the spiritual mind fuets physical wellness. Praegerl Greenwood, Westpon, CT

Plante TG (2009) Spiritual Practices in Psychotherapy: Thirteen Tools for Enhancing Psychological Health. American Psychological Association. Washington, DC

Powell L, Shahabi L, Thoresen CE (2003) Religion and spirituality: Linkages to physical health. Am Psychol 58: 36-52

Sloan RP, Bagiella E, Powell T (1999) Religion, spirituality, and medicine. Lancet 353: 664-7

\section{Critiquing Nursing Research 2nd Edition}

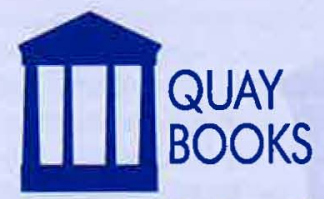

A division of MA Healthcare Ltd Quay Books is a leading independent publisher of nursing and medical texts with a continuing commitment to excellence.

\section{Critiquing} Nursing Research

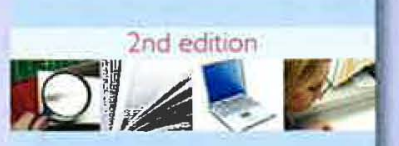

John Cutclife and Martin Ward

\section{By John R Cutcliffe and Martin Ward \\ ISBN-13: 978-1-85642-316-8; \\ ISBN-10: 1-85642-316-6, \\ $234 \times 156$ mm; phack; 244 pages;}

This 2 nd edition of Critiquing Nursing Research retains the features which made the original a 'best seller' whilst incorporating new material in order to expand the book's applicability. In addition to reviewing and subsequently updating the material of the original text, the authors have added two further examples text, the authors have added two further examples of approaches to crtitique along with examples and as part of the work of preparing a dissertation. The fundamentals of the book however remain the same. It focuses specifically on critiquing nursing research; the increasing requirement for nurses to become conversant with research, understand its link with the use of evidence to underpin practice; and the the use of evidence to underpin practice; and the
movement towards becoming an evidence-based discipline. As nurse education around the world increasingly moves towards an all-graduate discipline, it is vital for nurses to have the ability to critique research in order to benefit practice. This book is the perfect tool for those seeking to gain or develop precisely that skill and is a must-have for all students precisely that skill and is a must-h
nurses, teachers and academics.

wohn Cutcisfo holds the 'Davd Q. Bnithwa:e' Prolessar of Nursing Endowed Char at vo Unversity of Texas (Tyer); ne is also an Adjunct Professor of Psychiamic Nursing at Stenberg Coliege International School of Nursing, Vancouver, Canada Martin Ward is an Independent Mental Health Nurse Consultan and Director of MW Professional Deveropment Lld.
ORDER FORM

\section{QTY TITLE}

$\square$ Critiquing Nursing Research

2nd edition RRP \&25.99

To order your copy please contact us using the details above or visit our website

www.quaybooks.co.uk where you will also find details of other Quay Books offers and titles. 